\title{
A coordinated modeling assessment of the climate response to volcanic forcing
}

Davide Zanchettin', C. Timmreck², M. Khodri ${ }^{3}$, A. Robock ${ }^{4}$, A. Rubino' ${ }^{1}$ A. Schmidt ${ }^{5}$ and M. Toohey $y^{2,6}$

\section{Simulating volcanically-forced climate variability is a challenging task for climate models. The model intercomparison project on the climate response to volcanic forcing (VoIMIP) defines a protocol for idealized volcanic-perturbation experiments to improve comparability of results across different climate models.}

Stratospheric aerosols originating from volcanic sulfur emissions are a critical part of the natural forcing driving interannual-to-multidecadal climate evolution. In its 2013 assessment report, the Intergovernmental Panel on Climate Change (IPCC) affirms strong advances had been made in understanding volcanic aerosols and constraining associated forcing estimates compared with previous assessments (Myhre et al. 2013). Challenging the high confidence in volcanic forcing estimates reported by the IPCC (see Table 8.5 in Myhre et al. 2013), climate model results show some gaps in our understanding of the climate's response to volcanic eruptions. For example the largest uncertainties in the estimates of radiative forcing from historical simulations
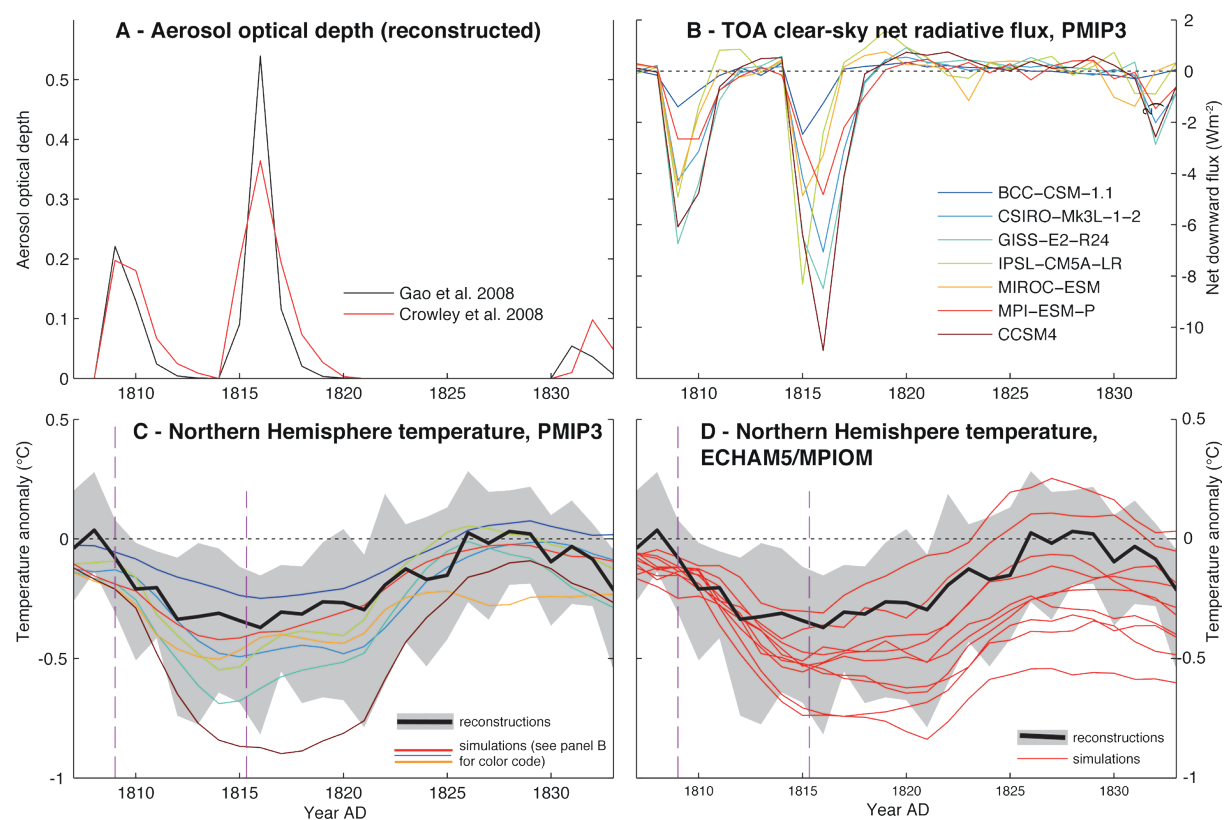

D - Northern Hemishpere temperature, ECHAM5/MPIOM

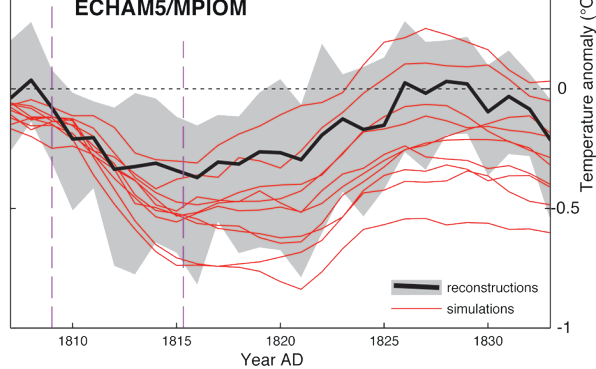

Figure 1: Uncertainty in radiative forcing and climate response for the early-19 $9^{\text {th }}$-century eruptions. (A) Two estimates of annual-average global aerosol optical depth at $550 \mathrm{~nm}$ (AOD). (B) Top-of-atmosphere (TOA) annualaverage net clear-sky radiative flux anomalies for a multi-model ensemble of last-millennium simulations (PMIP3; Braconnot et al. 2012). (C) Comparison between simulated (PMIP3, 11-year smoothing, colors) and reconstructed (black line: mean; shading: $5^{\text {th }}-95^{\text {th }}$ percentile range) Northern Hemisphere average summer temperature anomalies (from 1799-1808). (D) Same as (C), but for a single-model ensemble (ECHAM5/MPIOM; Zanchettin et al. 2013). The models tend to overestimate the reconstructed early-19th-century cooling, yet both simulation ensembles are compatible with the reconstruction; different models and forcing inputs (C) and internal climate variability (D) similarly contribute to simulation-ensemble spread. of uncertainty, such as the imposed volcanic forcing. The lack of agreement between model results is mainly due to differences in the model's characteristics, such as spatial resolution and implementation of volcanic forcing (e.g. Timmreck 2012). Nevertheless, whereas recent volcanic eruptions have been well observed and forcing estimates relatively well constrained, uncertainties grow considerably for events that occurred in the more remote past. For such eruptions, which contribute substantially to our understanding given the few instrumentally observed events, forcing characteristics must be reconstructed based on indirect evidence. This implies a lack of detail and large uncertainties regarding the climatically relevant parameters related to the source (especially the magnitude of the eruption), and to the stratospheric aerosol properties such as spatial extent of the cloud, optical depth, and aerosol size distribution (e.g. Timmreck 2012). All these large uncertainties are reflected in the occasionally substantial inconsistencies between available volcanological datasets (Sigl et al. 2014). Furthermore, internal climate variability and the presence of other forcing factors contribute to determine which mechanisms are activated in response to individual events (e.g. Zanchettin et al. 2013). It is therefore difficult to constrain the simulated responses to eruptions that occurred before the instrumental period, for which knowledge about the background climate conditions is poor.

It is thus unsurprising that different simulations, either performed with different climate models or with the same climate model, and climate reconstructions tell different, often divergent stories about the post-eruption climate evolution. This occurred, for instance, in the case of the 1815 Tambora event, the largest-magnitude volcanic eruption of the past five centuries (Fig. 1).

\section{Coordinated model intercomparisons}

The continuing development of more accurate histories of past eruptions (e.g. Sigl et al. 2014) and more realistic volcanic forcing datasets (e.g. Arfeuille et al. 2013) promises to improve climate model simulations of 
past volcanic events. This alone, however, is not enough to discern the individual contributions to uncertainty from the bulk of the varied factors illustrated above. It is also necessary to frame modeling activities within standardized experiments designed to systematically tackle specific uncertainty factors.

The ongoing Model Intercomparison Project on the climatic response to Volcanic forcing (VolMIP) has defined a common protocol to subject coupled climate models to the same volcanic forcing, thus aiming for negligible across-model differences in the applied radiative forcing (e.g. Fig. 1b) in order to focus on the climate response. The coordinated experiments will assess the causes of acrossmodel spread linked to the different treatment of physical processes, and separate such model uncertainties from uncertainties in the forcing and internal variability.

VolMIP focuses on the simulated processes that determine two main aspects of climate response to large volcanic eruptions: (i) the immediate dynamical alteration of atmospheric circulation triggered by the volcanically-induced stratospheric thermal anomaly and the associated variations in regional near-surface responses; and (ii) the decadal-scale response of the oceanic thermohaline and gyre circulations and associated long-term changes in heat transport and ocean-atmosphere coupling.

\section{The VolMIP experiments}

VoIMIP defines a set of idealized volcanic perturbations based on historical eruptions. In this context, "idealized" means that the volcanic forcing is derived from radiation parameters of documented eruptions and the experiments do not include information about the actual climate conditions when these events occurred. The experiments are designed as ensemble simulations, with sets of initial climate states sampled from an unperturbed preindustrial simulation. By exploring very different initial conditions, VoIMIP aims to constrain the range of post-eruption evolutions that arise from the interplay between ongoing internal climate variability and the imposed radiative perturbation, thus clarifying how much an accurate knowledge of background climate conditions matters when reproducing past volcanic events. Specific attention is given to the concomitant phasing of two dominant modes of climate variability: the El NiñoSouthern Oscillation, the most important source of interannual climate variability, and the Atlantic meridional overturning circulation, a measure of the strength of the oceanic thermohaline circulation.

The proposed core and sensitivity experiments (Fig. 2) reflect VolMIP's twofold strategy:

- VolShort: a set of experiments focusing on the seasonal-to-interannual atmospheric response to a 1991 Pinatubo-like volcanic eruption. The goal is to discriminate between the uncertainties that are due to internal variability (within-model

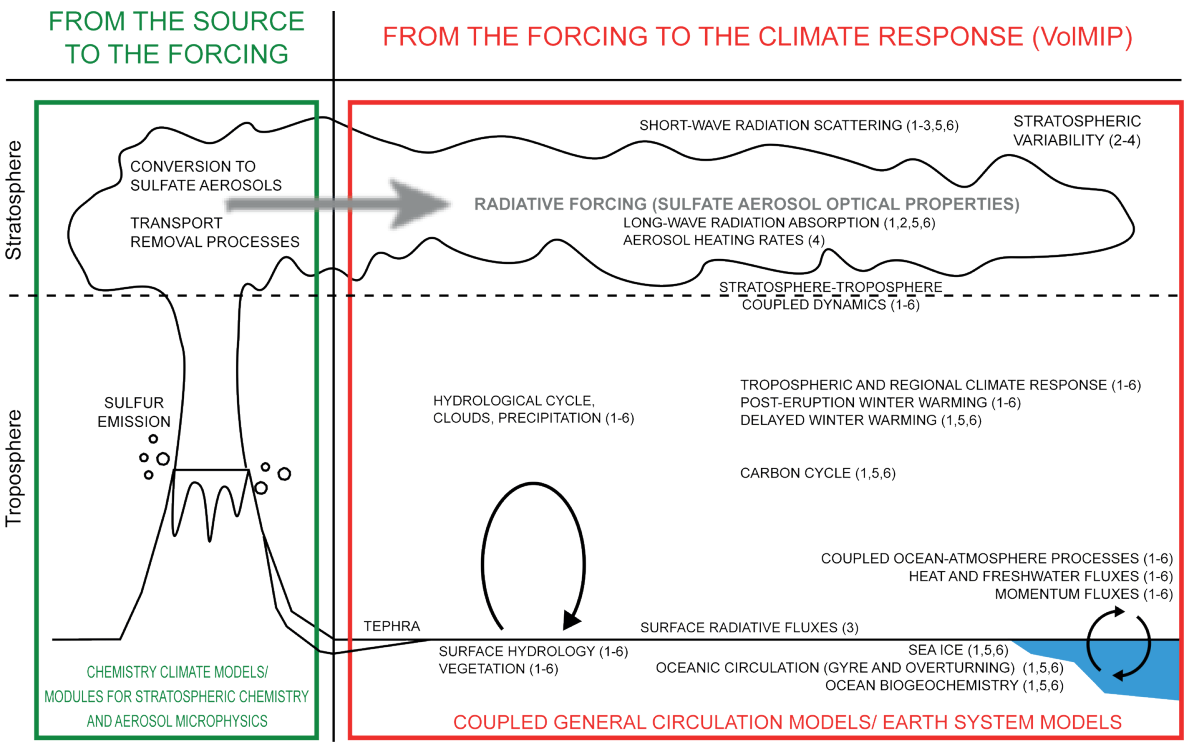

Figure 2: Dominant processes illustrating the link between volcanic eruptions and climate response, with an overview of VolMIP experiments as submitted to the World Climate Research Program for CMIP endorsement (1-4: mandatory; 5,6: non-mandatory). VoIMIP experiments: (1) Tambora-like tropical eruption [VolLongS60EQ]; (2) Pinatubo-like tropical eruption [VolShort20EQfull]; (3) Pinatubo-like eruption, prescribed perturbation to shortwave radiative flux [VolShort20EQsurf]; (4) Pinatubo-like eruption, prescribed perturbation to aerosol heating rates [VolShort20EQstrat]; (5) Laki-like high-latitude eruption [VolLongS100HL]; (6) 19 th $^{\text {century-like }}$ cluster of tropical eruptions [VolLongC19thC]

spread) and those due to model characteristics (across-model spread). The core full-forcing experiment is supported by sensitivity experiments designed to determine the roles of surface cooling and stratospheric warming - the two main features of short-term post-eruption climate variability - in controlling the dynamical response of the atmosphere. Additional experiments address the impact of volcanic forcing on seasonal-to-interannual climate predictability.

- VolLong: a set of experiments addressing the long-term (up to the decadal time scale) climate response to volcanic eruptions featuring a high signal-tonoise ratio in the global-average surface temperature response. Focus is on the signal propagation pathways of volcanic perturbations within the coupled atmosphere-ocean system, the associated determinant dynamical processes and their representation across models. The 1815 Tambora eruption is chosen as reference for the core experiment, as available climate-proxy data provide information on both eruption characteristics and climate response. A 1783 Laki-like, high-latitude eruption and idealized volcanic clusters (close successions of large volcanic eruptions) are also contemplated in this set of experiments. Provision of forcing input data for these simulations is an integral part of VoIMIP.

\section{Conclusions}

Improvement in understanding the dominant mechanisms behind simulated post-eruption climate evolution crucially depends on coordinated modeling activities that address the individual sources of uncertainty separately. By subjecting different models to well-constrained volcanic forcing, VolMIP promises to make significant progress in our knowledge of the physical processes that determine the climate's response to volcanic forcing. By further clarifying the relative role of internal and externally-forced climate variability during periods of strong radiative forcing, VoIMIP can enhance our ability to accurately simulate past, as well as future, climates.

\section{AFFILIATIONS}

'Department of Environmental Sciences, Informatics and Statistics, University of Venice, Italy ${ }^{2}$ Max-Planck-Institute for Meteorology, Hamburg, Germany

${ }^{3}$ IRD/IPSL/Laboratoire d'Océanographie et du Climat, Paris, France

${ }^{4}$ Department of Environmental Sciences, Rutgers University, New Brunswick, USA

${ }^{5}$ School of Earth and Environment, University of Leeds, UK

${ }^{6}$ GEOMAR Helmholtz Centre for Ocean Research Kiel, Germany

\section{CONTACT}

Davide Zanchettin: davide.zanchettin@unive.it

\section{REFERENCES}

Anchukaitis Ket al. (2012) Nat Geosci 5: 836-837

Arfeuille F et al. (2013) Atmos Chem Phys 13: 11221-11234 Braconnot P et al. (2012) Nature Clim Change 2: 417-424 Crowley TJ et al. (2008) PAGES News 16: 22-23 Ding Y et al. (2014) J Geophys Res 119: 5622-5637

Driscoll S et al. (2012) J Geophys Res 117, doi:10.1029/2012JD017607

Frank DC et al. (2010) Nature 463: 527-530

Gao C et al (2008) J Geophys Res 113, doi:10.1029/2008JD010239

Mann ME et al. (2012) Nature Geosci 5: 202-205

Marotzke J, Forster P (2015) Nature 517: 565-570

Myhre G et al. (2013) In: Stocker TF et al. (Eds) Climate Change 2013: The Physical Science Basis. Cambridge University Press, 659-740

Santer BD et al. (2014) Nature Geosci 7: 185-189 Sigl M et al. (2014) Nature Clim Change 4: 693-697

Timmreck C (2012) Wiley Inter Rev: Clim Change: 545-564 Zanchettin D et al. (2013) J Geophys Res 118: 4090-4106 